\title{
Repair of Spontaneous Cerebrospinal Fluid Otorrhea from Defect of Middle Cranial Fossa
}

\author{
Sung Hyun Boo ${ }^{1}$, Young Bum Goh ${ }^{1}$ and Chi-Sung Han ${ }^{2}$ \\ ${ }^{1}$ Department of Otorhinolaryngology-Head and Neck Surgery, Samsung Changwon Hospital, \\ Sungkyunkwan University School of Medicine, Changwon, \\ ${ }^{2}$ Ulsan Hana ENT Hospital, Ulsan, Korea
}

Received September 8, 2013

Revised October 28, 2013

Accepted November 12, 2013

\section{Address for correspondence Sung Hyun Boo, MD \\ Department of Otorhinolaryngology- Head and Neck Surgery, Samsung Changwon Hospital, Sungkyunkwan University School of Medicine, 158 Paryong-ro, Masanhoewon-gu, Changwon 630-522, Korea \\ Tel $+82-55-290-6563$ \\ Fax $+82-55-290-6465$ \\ E-mail descent@skku.edu}

Spontaneous cerebrospinal fluid (CSF) otorrhea is defined as CSF otorrhea where there are no identifiable causes including previous trauma, surgery, infection, neoplasm or congenital anomaly. The condition is rare. The origin of CSF leak is commonly a defect in the tegmen of the middle cranial fossa. The pathophysiology of spontaneous CSF otorrhea is unclear. Two theories of the etiology of bony defects of the temporal bone are the congenital bony defect theory and arachnoid granulation theory. The authors experienced a case of a 49-year-old female patient admitted with the complaint of persistent right ear fullness. Computed tomography revealed a large defect of the middle fossa and suspicious CSF otorrhea through the defect of tegmen tympani. Repair was successful with multiple bone chips using the transmastoid approach. The postoperative course was good and there has been no recurrence of the CSF leakage.

Korean J Audiol 2013;17:148-151

\section{Introduction}

Cerebrospinal fluid (CSF) otorrhea most commonly occurs as a result of trauma, surgery, or congenital anomalies. Although spontaneous CSF otorrhea is a rare condition, it is lifethreatening because of the high incidence of subsequent meningitis. Immediate and definitive repair of a CSF leak is important to prevent this potential complication. Surgical approaches include a transmastoid approach, middle fossa craniotomy, or a combined approach. We recently experienced the case of a patient with spontaneous CSF otorrhea occurring through a bony defect in the anterior wall and roof of the epitympanum. The defect was successfully repaired using bone chips with fascia by the transmastoid approach.

This is an Open Access article distributed under the terms of the Creative Commons Attribution Non-Commercial License (http://creativecommons. org/licenses/by-nc/3.0/) which permits unrestricted non-commercial use, distribution, and reproduction in any medium, provided the original work is properly cited.

\section{Case Report}

A 49-year-old woman was referred to our hospital because of right pulsatile otorrhea after myringotomy. Initially, she had experienced right ear fullness and hearing disturbance for 2 weeks. When a local otolaryngologist examined her ear, there was visible fluid through the tympanic membrane. Thus, she was diagnosed as right serous otitis media and treated with antibiotics. However, the fluid remained despite a 2 -week treatment. Myringotomy was attempted but failed due to profuse pulsatile clear discharge from the myringotomy site.

The pure tone audiogram of the right ear revealed conductive hearing loss of $40 \mathrm{~dB}$ (air-bone gap $32 \mathrm{~dB}$ ). Her body weight was $70 \mathrm{~kg}$ and body mass index was $27.7 \mathrm{~kg} / \mathrm{m}^{2}$. She had no specific past medical history and family history. High resolution computed tomography (HRCT) scan and magnetic resonance imaging (MRI) of temporal bone were performed for further evaluation of the otorrhea. The HRCT scan revealed a large, bony, anteromedial defect from the anterior wall to the roof of the right epitympanum (Fig. 1). The right middle ear cavity and mastoid cavity were filled with soft tissue density. 
T2 weighted MRI demonstrated high signal intensity in right middle ear and mastoid cavity, consistent with CSF (Fig. 2).

Repair of CSF leak was planned through a transmastoid approach. First, lumbar drainage was done after general anesthesia. Temporalis muscle fascia was harvested. Several pieces of bone chips were taken from mastoid cortex. A cortical mastoidectomy was performed. On entering the antrum, a profuse clear discharge from epitympanum was noted. To further evaluate the tegmen tympani, the incus and head of malleus were removed. An approximately $8 \mathrm{~mm}$ bony defect was apparent from the anterior roof of the epitympanum extending to the anterior wall. There was no identifiable any healthy dura or brain tissue herniation along with the CSF leak site.

Covering the defect began with insertion of prepared temporalis fascia into the bony defect. After that, large piece of bone chip was inserted into the defect to cover the fascia. The bony

Fig. 1. Preoperative HRCT scans of temporal bone. Axial image (A) demonstrates a large bony defect of the right anterior wall of the epitympanum (white arrow). Coronal image (B) shows a bony defect of the right middle cranial fossa. Also, tympanic and mastoid cavities are filled with soft tissue density. HRCT: high resolution computed tomography.
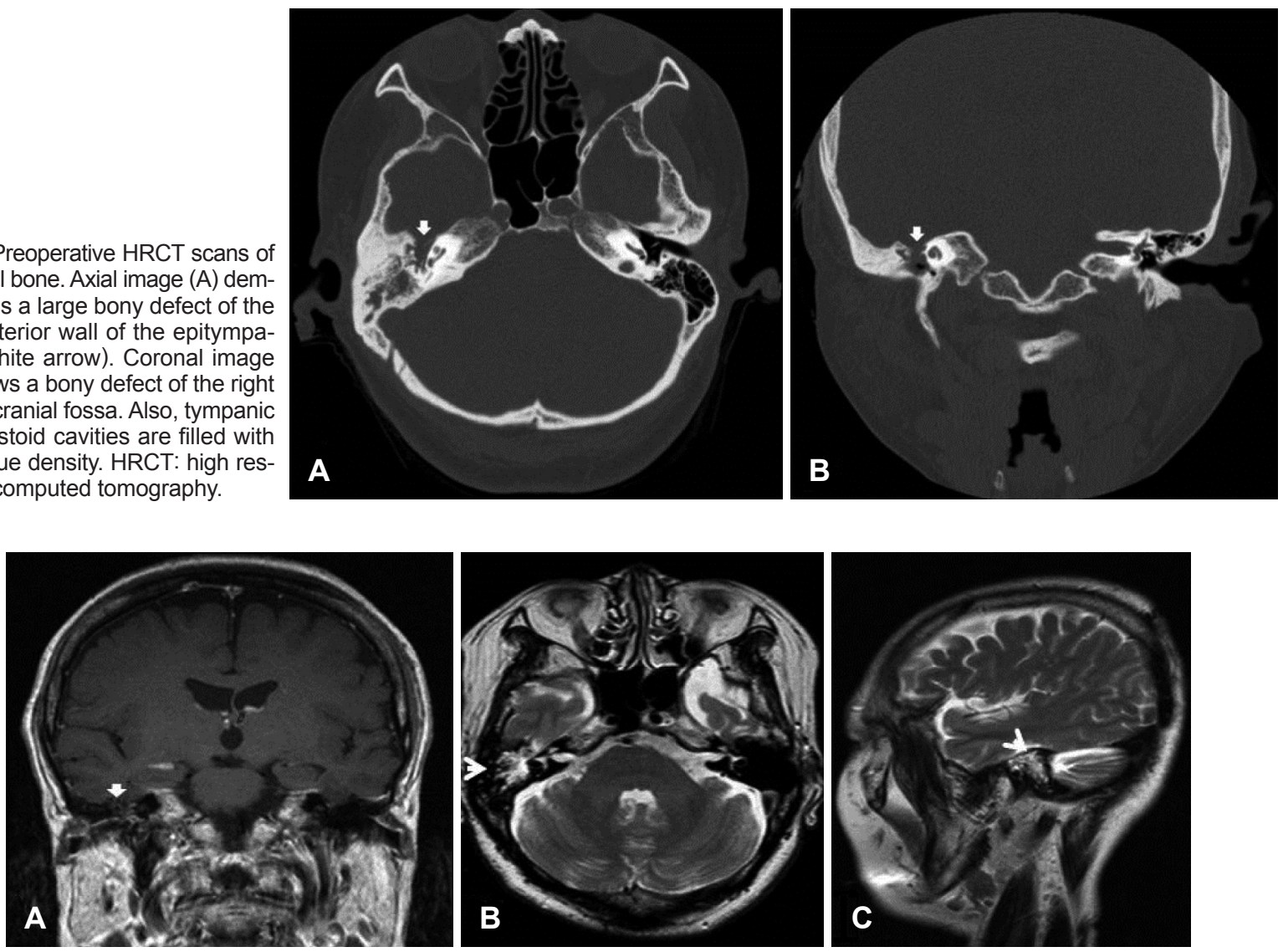

Fig. 2. Preoperative brain MRI scan results. Gd enhanced T1-weighted coronal image (A) shows no evidence of brain herniation in epitympanum and isosignal intensity (arrow). Unenhanced T2-weighted axial (B) and sagittal (C) image demonstrate high signal intensity in right middle ear and mastoid cavity (arrow head).

Fig. 3. Postoperative 1 week HRCT of temporal bone. Axial (A) and coronal (B) images show bone chips graft covering defect of right middle cranial fossa (white arrow). Pneumocephalus was noted in right temporal lobe (black arrow). HRCT: high resolution computed tomography.
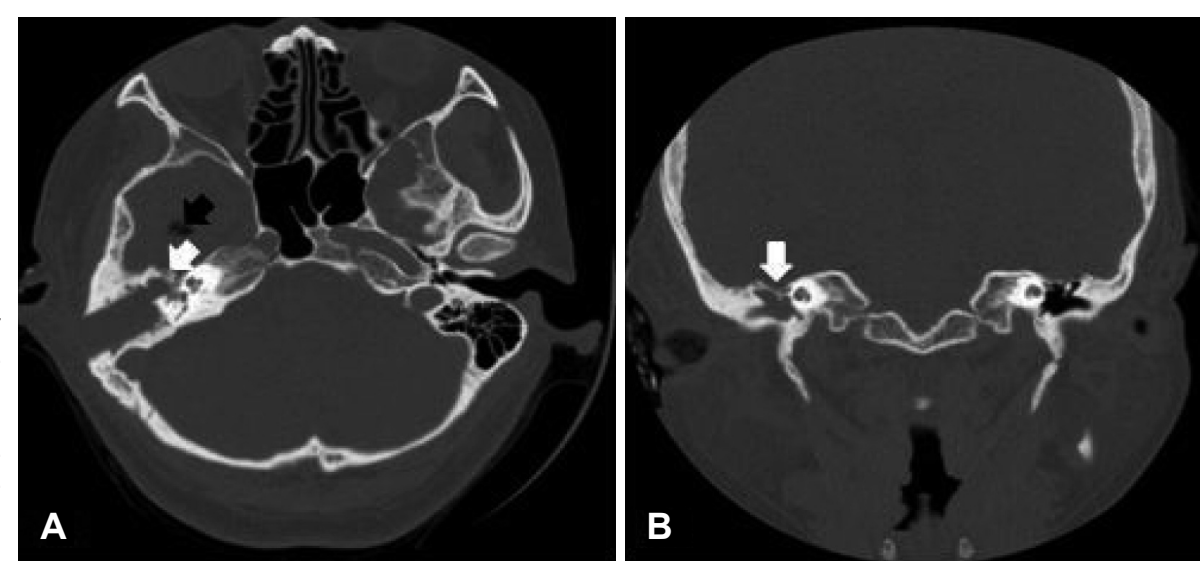

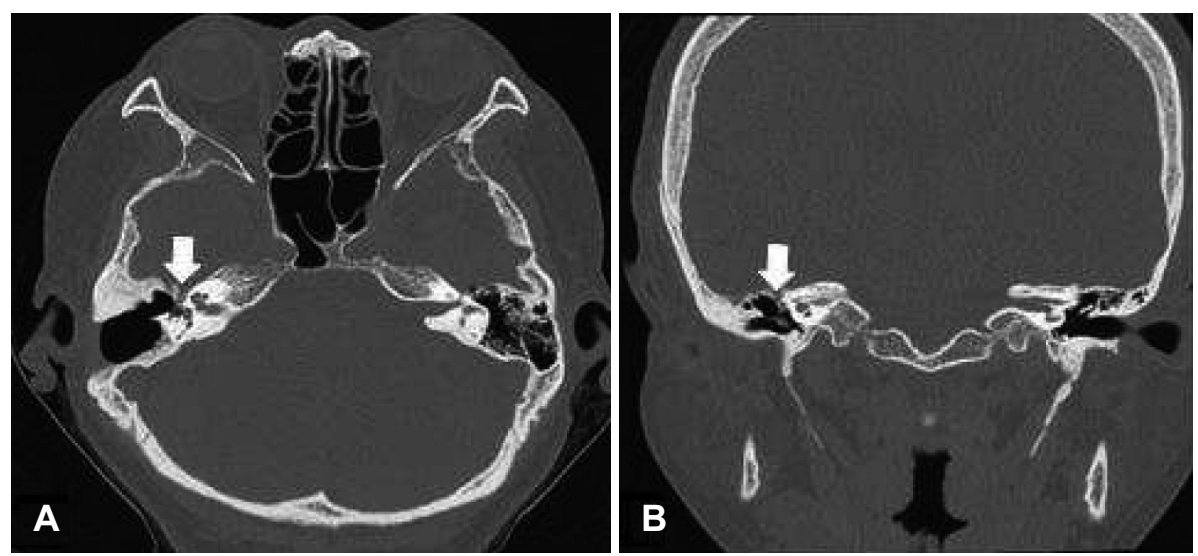

Fig. 4. Postoperative 1-year HRCT scan of temporal bone. Axial (A) and coronal (B) images demonstrate sustained anterior wall and roof of the epitympanum (white arrow). There is no evidence of CSF leak in the tympanic cavity. HRCT: high resolution computed tomography, CSF: cerebrospinal fluid.

defect was still visible because of the large defect size. Other bone chips were placed in the defect, unless there was no evidence of the CSF leak. Surgical glue was applied around bone chips and gelfoams were packed in the cavity.

A postoperative CT scan demonstrated that the defect had sealed and revealed a pneumocephalus in the right temporal lobe (Fig. 3). The patient did not complain of headache or any neurologic deficits. Lumbar drainage was maintained for 6 days. Two weeks later, the pneumocephalus had disappeared and the patient was discharged. One year later, CT scan showed a well-healed defect (Fig. 4). There has been no evidence of recurrent CSF leak up to date.

\section{Discussion}

Spontaneous CSF otorrhea is defined when there are no identifiable causes such as past history of trauma, surgery, infection or tumor. ${ }^{1)}$ The origin of the CSF leak is commonly a defect in the tegmen of the middle cranial fossa, less in posterior fossa.

The pathophysiology of spontaneous CSF otorrhea is not entirely clear. There are two main theories concerning the etiology of bony defect. The first is the congenital defect theory, which posits that tiny defects within the tegmen caused by aberrant embryologic development enlarge over time secondary to constant CSF pressure. This enlargement leads to eventual dural herniation and subsequent bony and dural thinning with resultant CSF otorrhea. ${ }^{2)}$ The arachnoid granulation theory postulates that arachnoid granulations that do not find a venous termination during embryonic development come to lie in a blind-end against the inner bony surface of the skull. ${ }^{3)}$ These granulations enlarge with age and may eventually erode bone. Aberrant arachnoid granulations located in the dural surface of the temporal bone are thought to be responsible for communication between the CSF space and the mastoid air cell system. Gacek ${ }^{3)}$ identified an $8.5 \%$ incidence of arachnoid granulations in the posterior fossa of the temporal bone, while Ferguson, et al. ${ }^{4)}$ observed an incidence of $22 \%$ of pit holes created by arachnoid granulations in the middle fossa surface.

Recent clinical studies have drawn attention to demographic features of patients with spontaneous CSF otorrhea as potential clues to the cause. These include age, low grade inflammation, and increased intracranial pressure (ICP). ${ }^{2)}$ Females are affected more often than males. ${ }^{5)}$ Middle-aged and obese (body mass index $>30 \mathrm{~kg} / \mathrm{m}^{2}$ ) persons are more apt to develop spontaneous CSF otorrhea. ${ }^{6,7)}$ Schlosser, et al.$^{8)}$ reported increased ICP and idiopathic intracranial hypertension as common findings among patients of spontaneous CSF otorrhea and suggested that obesity and increased BMI may play a role. The subject of this case was also an obese, middle-aged woman.

Bone defect of cranial fossa can be closed using a variety of autologous materials, such as fascia, fat, muscle, cartilage, and bone, or synthetic materials including silicone, silastic, Marlex, hydroxyapatite cement, and titanium plate. ${ }^{9)}$ Each material must be strong enough to withstand intracranial pressure and be compliant enough to form a seal.

There are three surgical approaches for the repair of the CSF otorrhea: transmastoid approach, middle fossa craniotomy, and a combined approach. ${ }^{3,9,10)}$ Surgeons can choose between these options based on the location and size of defect, their experience or preference, and better surgical view. The favored technique is a combined transmastoid-middle fossa approach, which visualizes the entire tegmen and definite closure of the entire region. But, this approach has a great potential for complications and should be performed by experienced operators. In contrast, the transmastoid approach is technically easier to perform, has fewer risks and complications, and anterior defect in the tegmen tympani may require the removal of ossicles to ensure better exposure of the lesion. It causes hearing disturbance that can be restored with ossicular reconstruction in the same or second stage. In this case, the lesion is located anteromedial side of middle cranial fossa so the combined ap- 
proach could be suitable. However, we could perform in transmastoid approach with only of removal of ossicles to obtain clear view of lesion and successfully manage the defect.

\section{REFERENCES}

1) Jahrsdoerfer RA, Richtsmeier WJ, Cantrell RW. Spontaneous CSF otorrhea. Arch Otolaryngol 1981;107:257-61.

2) Pappas DG Jr, Hoffman RA, Cohen NL, Pappas DG Sr. Spontaneous temporal bone cerebrospinal fluid leak. Am J Otol 1992;13:534-9.

3) Gacek RR. Arachnoid granulation cerebrospinal fluid otorrhea. Ann Otol Rhinol Laryngol 1990;99:854-62.

4) Ferguson BJ, Wilkins RH, Hudson W, Farmer J Jr. Spontaneous CSF otorrhea from tegmen and posterior fossa defects. Laryngoscope 1986;96:635-44.

5) Gubbels SP, Selden NR, Delashaw JB Jr, McMenomey SO. Sponta- neous middle fossa encephalocele and cerebrospinal fluid leakage: diagnosis and management. Otol Neurotol 2007;28:1131-9.

6) LeVay AJ, Kveton JF. Relationship between obesity, obstructive sleep apnea, and spontaneous cerebrospinal fluid otorrhea. Laryngoscope 2008;118:275-8.

7) Goddard JC, Meyer T, Nguyen S, Lambert PR. New considerations in the cause of spontaneous cerebrospinal fluid otorrhea. Otol Neurotol 2010;31:940-5.

8) Schlosser RJ, Woodworth BA, Wilensky EM, Grady MS, Bolger WE. Spontaneous cerebrospinal fluid leaks: a variant of benign intracranial hypertension. Ann Otol Rhinol Laryngol 2006;115:495-500.

9) Kutz JW Jr, Husain IA, Isaacson B, Roland PS. Management of spontaneous cerebrospinal fluid otorrhea. Laryngoscope 2008;118: 2195-9.

10) Bento RF, Pádua FG. Tegmen tympani cerebrospinal fluid leak repair. Acta Otolaryngol 2004;124:443-8. 